\title{
Structural optimization of high voltage transmission line towers considering continuum and discrete design variables
}

\author{
J. París, S. Martínez, F. Navarrina, I. Colominas \& M. Casteleiro \\ GMNI - Group of Numerical Methods in Engineering, \\ Civil Engineering School, University of A Coruña, Spain
}

\begin{abstract}
Structural optimization has been usually applied to singular projects (e.g. a dam) and/or to common designs largely repeated (e.g. automotive components), and high voltage transmission towers can be included in both groups since they are expensive and a large number of them is required.

In this paper, the authors propose a general formulation for obtaining the structural optimum design of latticed high voltage towers. The formulation is devoted to obtaining the most common objective in engineering (minimum cost) considering the limitations imposed in actual norms for this kind of structure. According to this idea, real applications are studied and modeled under real conditions (e.g. loads, constraints, building process). Constructive aspects like the specific geometry, the structural elements or the building process are specifically considered. The optimization model proposed also deals with continuum design variables (global geometry variables) and discrete design variables (area, inertia and/or cross section dimensions, for example) both together. The use of both types of design variables is crucial for defining a realistic model that can be used in practical applications in engineering. The optimum design formulation proposed is general and can be easily applied to other different types of 3D latticed structures.

Finally, a real application example of a high voltage tower under real conditions and requirements is analyzed.

Keywords: structural optimization, high voltage towers, discrete design variables, continuum design variables.
\end{abstract}




\section{Introduction}

Structural design is a crucial topic in engineering since it allows to propose solutions to multiple challenges in modern societies. Design process has been continuously studied and applied in real problems in engineering and many other disciplines. However, this kind of procedures to propose adequate designs has become insufficient nowadays. Design methods need to be reformulated by including optimization techniques since traditional designs are usually effective but they are not the most efficient, in general.

In this paper the authors propose an optimization methodology that allows to obtain more efficient solutions than conventional designs of high voltage transmission line towers. The optimization model proposed was developed by taking into account specific aspects related to this kind of structures in order to give a realistic approach of the problem. In addition, these practical considerations facilitate the transference of optimized designs from numerical models to industrial applications. According to this idea, the proposed formulation tries to keep the main considerations related to the building process and includes all the design specifications established in the current norms and standards for this kind of structures in Spain. The model proposed includes all the design constraints proposed in the Spanish and European Standards [1, 2], but other similar constraints that could appear in other norms and laws can be easily included [3, 4].

The formulation proposed allows to deal with continuum design variables (related to global geometric properties of the structure) and with discrete design variables (related to cross-section properties of the bars that define the structure) both together. According to this, the methodology proposed deals with the discrete set of commercially available steel rolled sections which are generally used to define the structure of high voltage transmission line towers. In addition, the proposed methodology gives an optimized solution with conventional computing resources in adequate, and perfectly affordable, CPU time.

Finally, a real application example is analyzed in order to test the complete methodology developed and to verify the validity of the results in practical application cases.

\section{Structural model}

In the introduction section the authors present the main characteristics of the optimization methodology developed for the design of high voltage towers and the practical considerations required to obtain adequate solutions. In real applications, most of this kind of structures correspond to three dimensional latticed structures assembled by joining a set of rolled steel sections. These steel bars usually correspond to symmetric L-shaped cross-sections according to a commercial catalog. In addition, the steel sections are usually bolted in real designs due to practical considerations during the building process. Nowadays there exist rare specific designs with welded joints but they are not commonly used in practice. Consequently, the structural model for this kind of towers corresponds 

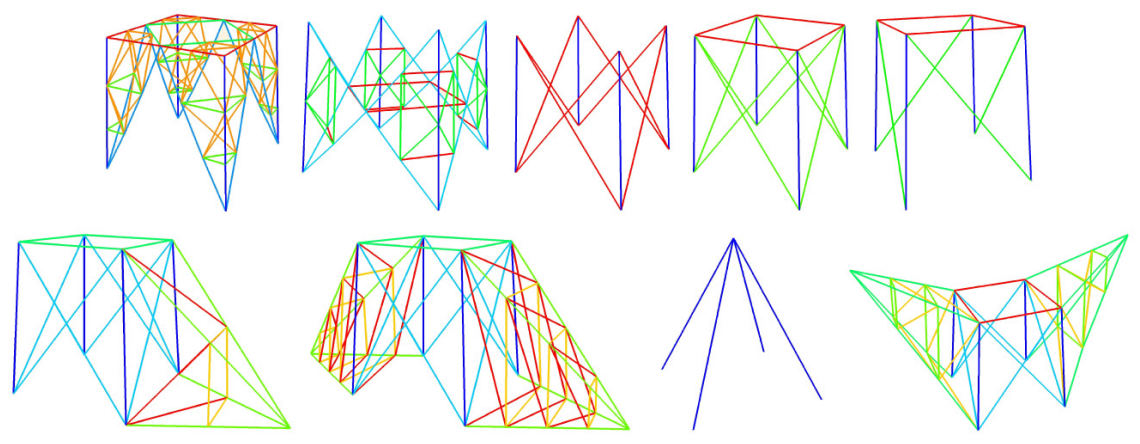

Figure 1: Examples of blocks commonly used in Spanish designs of high voltage towers.

to a 3D lattice of symmetric L-shaped cross-section bars with bolted joints. From a practical point of view, most of the joints of the structure correspond to articulations but there are some of them that correspond to semi-rigid articulations (specially in the vertical bars). From a mathematical point of view the numerical model used to develop the structural analysis corresponds to a 3D articulated bars formulation, since it adequately approximates the structural response. In addition, some important standards like the ones proposed by the ASCE $[3,4]$ also recommend this kind of numerical models.

Furthermore, high voltage transmission line towers usually have large dimensions (tens of meters high and some meters wide) and are built in practice by assembling a set of smaller blocks with predefined geometric and structural properties. Some of the most frequent blocks used in the Spanish and European towers can be observed in figure 1 .

The combination of some of these blocks, with adequate shape and dimensions, produces the final designs which are used in practice (figure 2).

In addition, it is important to take into account that for practical considerations the number of different rolled steel sections per block must be small. In practice, the symmetry of the design and the external forces applied suggest that the crosssection of a number of elements in a block (the vertical bars for instance) must be equal. Thus, each block is usually defined by adequately combining a small number of different rolled steel sections, depending on the complexity of the block. The distribution of different steel rolled sections can be also observed in figure 1 .

\section{Structural analysis}

The structural analysis model proposed in this paper corresponds to an articulated latticed structure made of predefined blocks and normalized steel rolled sections. Consequently, the numerical analysis model under the conventional hypotheses of small displacements, small displacements gradients and linear elasticity 

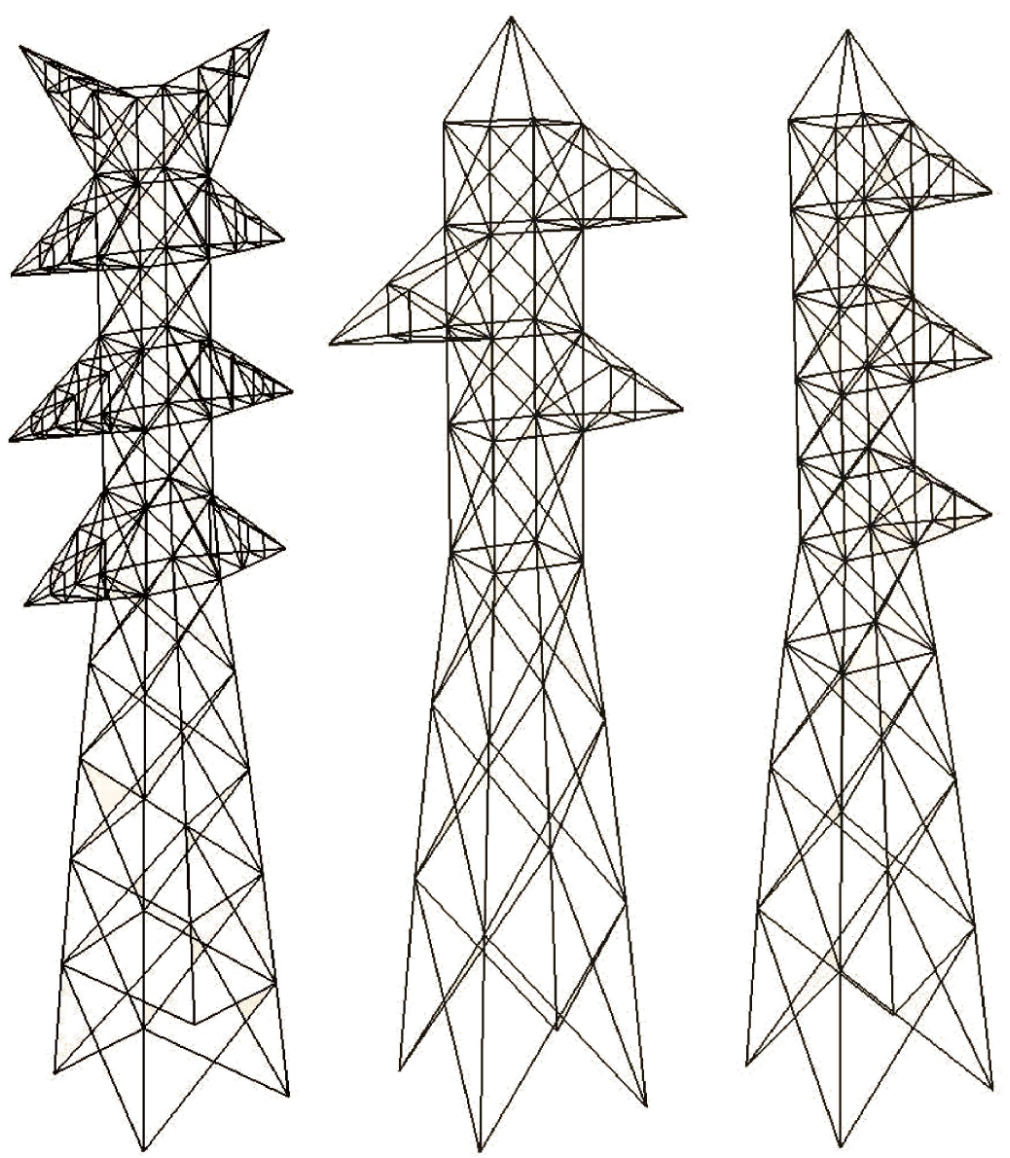

Figure 2: Examples of real designs of high voltage towers.

corresponds to a 3D analysis of articulated latticed structures. Furthermore, the Spanish and European Standards and Norms corresponding to this kind of structures $[1,2]$ impose a set of load cases and additional design constraints. The Spanish Standard [2] states that five different loads must be considered:

- Self weight of the tower and the conductors

- Ice load on the full length of the conductors

- Wind load on the tower and on the conductors

- Tension imbalance of the conductors connected to the tower on both sides along the line.

- Collapse of one conductor.

Figure 3 shows the displacements of a general design of a high voltage transmission line tower for the loads indicated in the actual Spanish Norm [2]. 

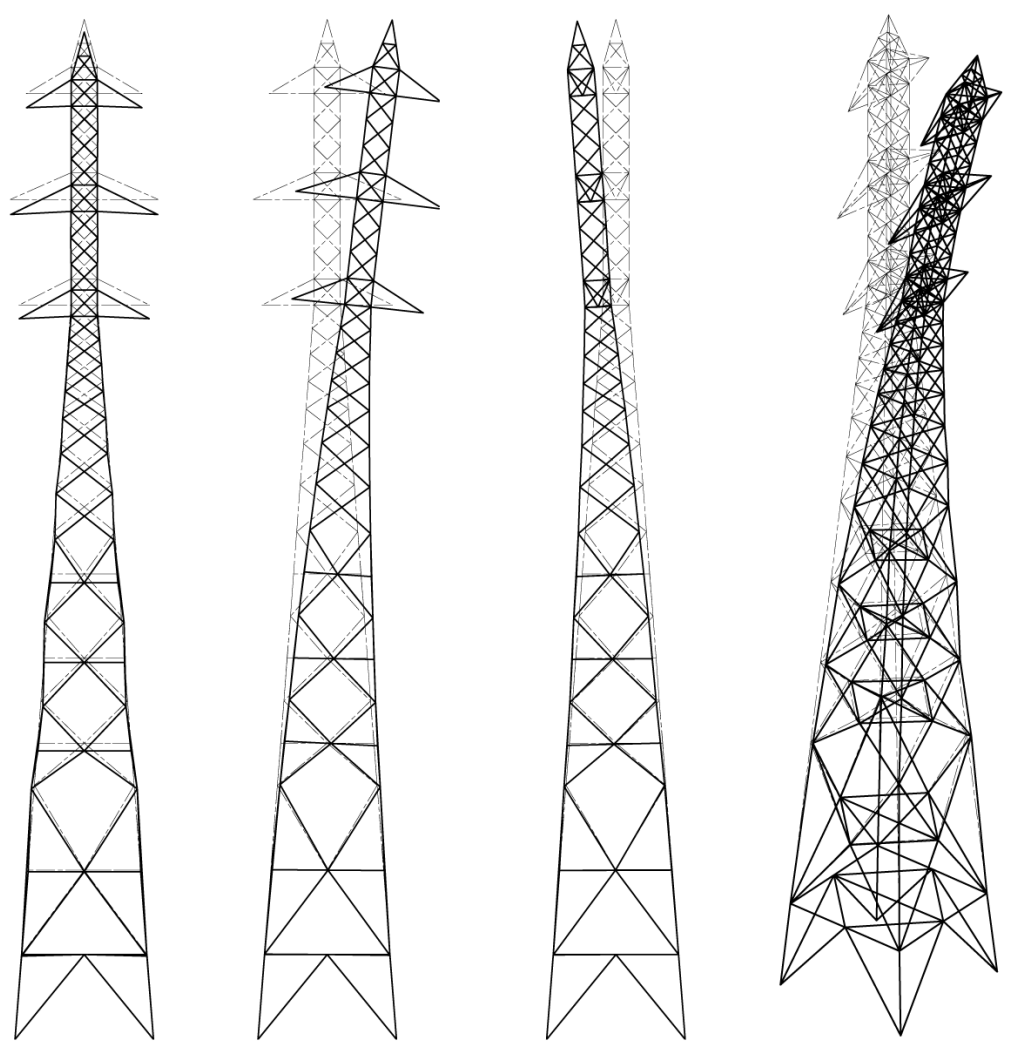

Figure 3: Displacements of a high voltage transmission line tower for (from left to right): ice load, wind load, tension imbalance of the conductors and collapse of one conductor.

The combination of these loads produces more than 7 different load cases. For each one of the indicated load cases, the structure must satisfy design constraints on:

- Elemental shape-section ratio: The section of the bars that define the structure corresponds to symmetric L-shaped sections. This kind of sections can be identified by the thickness and the length of their legs. The Spanish and European Standards [1, 2] impose a maximum value for the ratio between the length and the thickness of the cross-section legs in order to avoid local buckling.

- Elemental section-length ratio: The ratio between the radius of gyration of the cross-section and its length must satisfy a minimum value. This constraint is also related to buckling phenomena and it will be used further in the definition of other additional constraints. 
- Elemental tension limits: Each bar of the structure must satisfy the maximum tension stress of the material for each load case. Otherwise the structure will collapse.

- Elemental compression limits: Each bar of the structure must satisfy the minimum compression stress of the material being used for each load case. In addition, this constraint must guarantee that global buckling of bars do not appear. This buckling constraint is considered by taking into account the value of the section-length ratio proposed in the second item.

In practice, the proposed problem involves at least 7 load cases and each load case requires the verification of tension stress, compression stress and buckling constraints for each bar of the structure. In addition, geometrical constraints related to shape and dimensions of the bars need to be considered.

\section{Design model}

The structural model proposed in the previous section deals with realistic considerations that must be satisfied in order to accomplish with the Spanish and European Standards. The design model must allow to verify all these constraints and to improve the design without loosing any of the main properties of the conventional designs used in practice. Thus, the design model proposed must keep the modular construction by assembling blocks of predefined typologies vertically. In addition, the sections of the bars that define the structure must correspond to one of the normalized steel rolled sections defined in Spanish and European Standards and Norms due to economic and practical considerations. In addition, the multiple sections of the bars that define a block must be treated in groups according to their structural properties, as it can be observed in figure 1. Thus, the bars of each group present identical cross-sectional properties and dimensions.

According to these ideas the design model must include discrete design variables related to the cross-section properties of the groups of bars defined. These design variables can only take a set of predefined values established on normalized steel rolled sections catalogs. On the other hand, it is also important to allow the modification of the external geometry of the high voltage tower in order to improve the design. Consequently, the main dimensions of the blocks of the structure can be also included as design variables in the optimization problem. The formulation proposed includes the dimensions (width and length) of the upper and lower bases of the blocks as design variables. The heights of the blocks are treated as design constants in this paper. However, the inclusion of the heights of the blocks as design variables should not increase substantially the complexity of the underlying optimization problem. The geometrical design variables proposed can be included in the model as continuum variables since the length of each bar can be easily adjusted during the constructive process.

Thus, the design model proposed to optimize the structure of high voltage transmission line towers includes both continuum and discrete design variables. This fact introduces even much more complexity in the model than the large number of constraints and load cases presented in section 3. The use of both 
types of variables in one optimization model is a challenging problem nowadays, specially when realistic studies with a large number of design variables and constraints needs to be addressed. Conventional algorithms can not deal with discrete design variables and special algorithms that consider discrete variables are not efficient enough to manage a large number of discrete design variables and constraints. Specific algorithms like evolutionary methods or genetic algorithms have been used in the literature [5] but most of the practical considerations proposed in this paper were not stated. In practice most of the works related to this topic only study the discrete or the continuum problem. As far as the authors know both types of design variables (continuum and discrete) have not been previously treated in real applications related to the main objective of this paper.

\section{Optimization algorithm}

The optimization problem stated in the design model section presents crucial aspects that can not be treated with conventional optimization algorithms. According to that and considering previous works related to this topic [5-7], the authors propose an optimization methodology based on heuristic approaches. More specifically, the authors have developed a numerical model based on Simulated Annealing [8-13] since this formulation can include natively continuum and discrete design variables and it obtains optimal solutions for real cases in an acceptable computing time.

This algorithm is based on heuristic rules that allows to simulate the minimization problem defined in the previous sections by assuming that the optimization process is analogous to the cooling and annealing of metals in metallurgy. Furthermore, this formulation also allows to include design constraints. However, this optimization algorithm requires to compute and verify a large number of designs. The analysis of the structural problem and the verification of all the stress constraints is not critical from a computational point of view. However, the large number of designs to be analyzed turns the CPU time a critical aspect. Consequently, the authors have developed a numerical approximation of the objective function and the constraints based on first order Taylor expansions in order to reduce the required computing time. These Taylor expansions of the objective function and the design constraints allow to verify a large number of modified designs with a relatively small computing effort. On the other hand, first order derivatives of the objective function and the constraints needs to be computed. These derivatives are obtained analytically by using direct differentiation techniques $[6,7,14,15]$ in order to avoid propagation of rounding errors and to reduce the computing time required.

Design constraints are included in the optimization methodology by using penalty functions to define the objective function. In addition, normalized values of the objective function and the constraints are used in practice in order to avoid scale problems due to different natures of the objective function (the weight of the structure) and the design constraints (geometrical dimensions, stresses, ... ). The objective function is normalized by dividing the current weight over the initial 
weight, and design constraints are normalized by dividing its value (computed as the difference between the computed values and a established maximum) over the maximum value allowed. This formulation allows to obtain adequate sensitivities for applying Taylor expansions at each global iteration of the simulated annealing process. The use of Taylor expansions allows to drastically reduce the computing effort devoted to explore and study neighbor designs of a previous solution. In practice the computing time devoted to this exploration of the neighborhood can be reduced two orders of magnitude without loosing suitable precision in the computations.

However, it is obvious that first order Taylor expansions introduce considerable error if great modifications are applied. To avoid this effect, the modification of continuum variables is limited by using moving limits around the design solution previously obtained. The values for the discrete design variables are stated by searching adequate solutions on the neighborhood of the current design. This exploration is performed by considering increasing levels of neighborhood. First exploration tries to find an improved solution in the first set of contiguous values for each discrete design variable. If no adequate solution is found then a second level of neighborhood is also explored for the discrete design variables and so on. Moving limits of continuum design variables are also amplified. If no improvements are achieved after increasing the level of neighborhood up to a certain predefined limit, the search on discrete design variables stops since no better solution can be achieved.

\section{Application example}

The validity of the formulation presented in previous sections is developed by solving a real application example. In this paper, the authors present the solution obtained for the structural optimization of a $400 \mathrm{kV}$ transmission line tower with double circuit and two ground conductors on top. These towers are $41.20 \mathrm{~m}$ tall and present 7 different types of blocks, 66 groups of bars defined with normalized steel rolled sections and 32 continuum geometrical variables. In this case, the current Spanish Norm [2] imposes more than ten thousand design constraints that need to be considered during the optimization process.

Figure 4 (left) shows the initial geometry and materials for one of the actual designs commonly used in Spain in this kind of high voltage transmission lines. Figure 4 (right) shows the final optimized design obtained by using the methodology proposed in this paper. The use of the optimization formulation allows to reduce $25.7 \%$ of the initial weight of an actual design without modifying neither the topology nor the constructive process.

\section{Conclusions}

In this paper the authors develop an entire optimization methodology that allows to minimize the weight of high voltage transmission line towers by satisfying all 

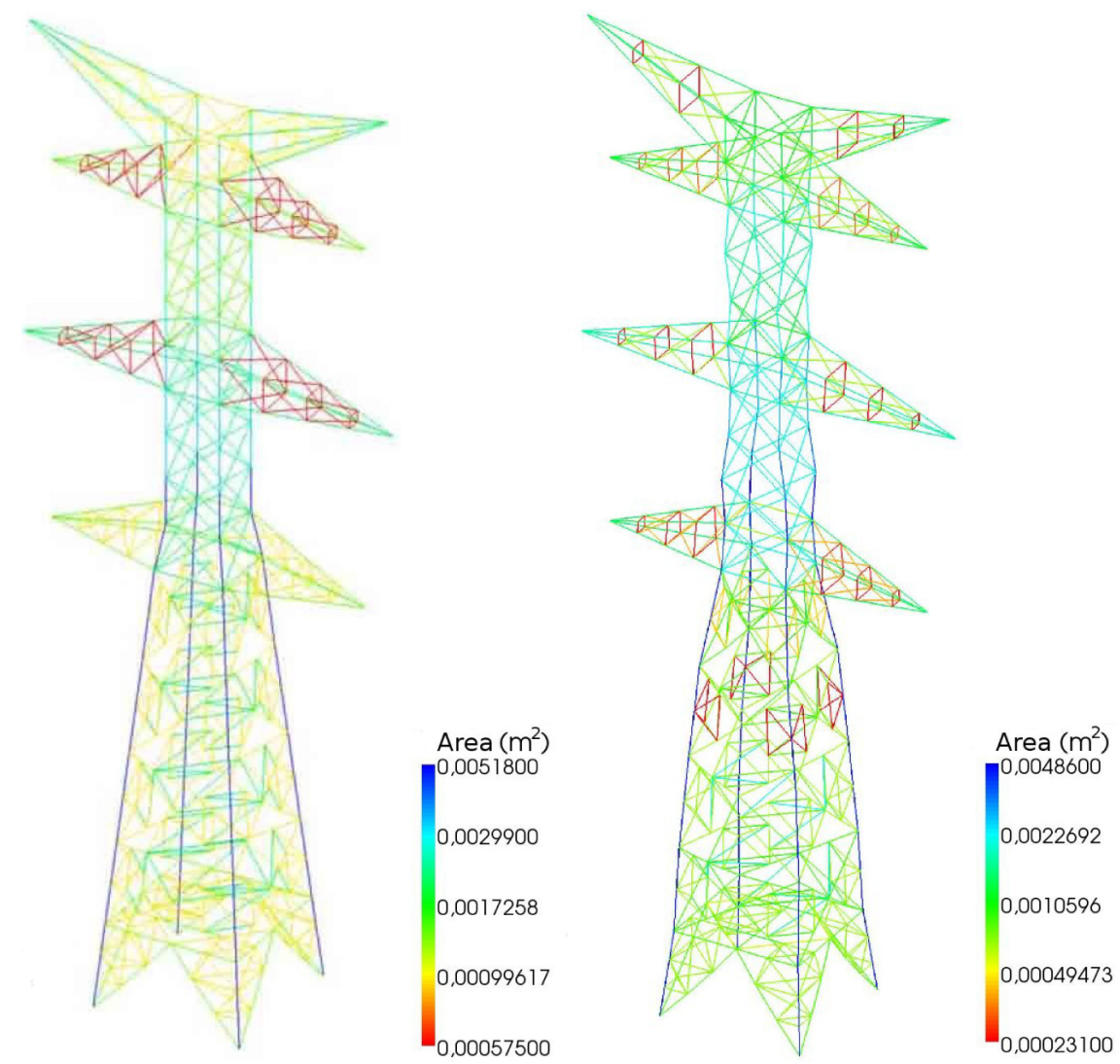

Figure 4: Initial design of the high voltage tower (left) and final optimized design (right).

the requirements imposed in the European and Spanish Standards and Norms $[1,2]$. The formulation proposed is realistic and it considers all the practical specifications required during the constructive process. The optimal designs obtained satisfy all the specifications included in the actual norms and can be directly applied in practice since they satisfy all the safety conditions imposed. According to this idea, real structural models are analyzed by using 3D articulated latticed structure models. These models are defined by assembling a set of predefined blocks according to the conventional constructive process.

In addition, the optimization model allows to deal with continuum design variables that define the global geometry of the structure and discrete design variables that correspond to the set of commercially available rolled steel sections. Consequently, the optimization problem can not be treated with conventional optimization algorithms. 
The proposed formulation allows to obtain important material savings in real application examples in an acceptable computing time.

\section{Acknowledgements}

This research work has been partially supported by the "Ministerio de Ciencia e Innovación" of the Spanish Government (DPI2009-14546-C02-01 and DPI201016496), by the Autonomous Government of the "Xunta de Galicia" (Grants CEOU 2007/09, PGDIT09MDS00718PR and PGDIT09REM005118PR) cofinanced with FEDER funds, by the "Universidade da Coruña" and by the "Fundación de la Ingeniería Civil de Galicia".

\section{References}

[1] European Committee for Standardization, EN 1993 - Eurocode 3: Design of steel structures, 1994.

[2] Ministerio de Industria, Turismo y Comercio (Spanish Ministry of industry, tourism and trade), Reglamento sobre condiciones técnicas y garantías de seguridad en líneas eléctricas de alta tensión y sus instrucciones técnicas complementarias ITC-LAT 01 a 09 (Spanish Standard about high voltage transmission lines), Boletín Oficial del Estado del 15 de Febrero de 2008, pp. 16436-16554, 2008.

[3] American Society of Civil Engineers, ASCE 1097: Design of Latticed Steel Transmission Structures, 1997.

[4] American Society of Civil Engineers, ASCE Manual 52: Design of Steel Transmission Towers, 1988.

[5] Vieswara Rao G., Optimum Design for Transmission Line Towers, Computers and Structures, Vol. 57(1): 81-91, 1995.

[6] Navarrina F., Valera A., París J., Colominas I., Casteleiro M., Optimization of High Tension Towers by Sequential Linear Programming with Quadratic Line Search, Proceedings of the Ninth International Conference on Computer Aided Optimum Design in Engineering, Skiathos, Grecia, 2005.

[7] París J., Martínez S., Navarrina F., Colominas I., Casteleiro M., Structural Optimization of High Tension Towers, Proceedings of the $2^{\text {nd }}$ International Conference on Engineering Optimization, Lisbon, Portugal, September 6-9, 2010.

[8] Kirkpatrick S., Gelat C. D., Vecchi M.P., Optimization by Simulated Annealing, Science, New Series, Vol. 220(4598):671-680, 1983.

[9] Ledesma S., Aviña G., Sánchez R., Practical Considerations for Simulated Annealing Implementation, Simulated Annealing, Vol. 20:401-420, 2008.

[10] Lundy M., Mees A., Convergence of an Annealing Algorithm, Mathematical Programming, Vol. 34:111-124, 1986.

[11] Wah B. W., Chen Y., Wang T., Theory and Applications of Simulated Annealing for Nonlinear Constrained Optimization, Simulated Annealing, 9:155-187, 2008. 
[12] White S. R., Concepts of Scale in Simulated Annealing, Proceedings of the IEEE International Conference on Computer Design, Port Chester, USA, pp. 646-651, 1984.

[13] Cruz J. R., Dorea C. C. Y., Simple Conditions for the Convergence of Simulated Annealing Type Algorithms, Journal of Applied Probability, Vol. 35(4): 885-892, 1998.

[14] Schmit L. A., Structural design by systematic synthesis, Second Conference on Electronic Computation, Pittsburg, USA, pp. 105-132, 1960.

[15] Choi K. K., Kim N., Structural Sensitivity Analysis and Optimization, Mechanical Engineering Series, Springer, 2004. 